\title{
Formación investigativa para la educación superior desde una perspectiva pedagógica
}

Investigative training for higher education from an educational perspective

Artículo recibido: mayo de 2010 Artículo aprobado: marzo de 2011
Hamlet Santiago González Melo*

Reflexionar en torno a la función de Investigación de la Universidad es hacerlo acerca de su esencia misma.

Ciro Parra Moreno

\section{Resumen}

En el presente artículo de reflexión se plantea la importancia de la investigación en la educación superior, en especial el papel que ocupa la investigación formativa en los procesos de enseñanza en la universidad.

\section{Palabras clave}

Universidad, conocimiento, investigación, docencia universitaria, investigación formativa.

\begin{abstract}
This reflexive article presents the importance of formative reserch in higher education partucularly the role that ocuupies the formative research in the processes of teaching at the University.
\end{abstract}

\section{Key words}

University, investigation, knowledge, teaching at universities, formative investigation.

\section{Introducción}

Abordar de una manera extensa la temática en cuestión implicaría realizar un recorrido histórico-analítico sobre la relación entre investigación y educación superior en la sociedad Colombiana, revisar la constitución de los currículos de diferentes facultades, departamentos y programas, teniendo en cuenta el lugar que se le ha asignado al componente investigativo, así como analizar la abrupta aparición del término "investigación" en la enunciación de las políticas educativas, manteniendo presente las múltiples posibilidades de interpretación asociadas a este concepto.

Profesor en la Facultad de Ciencias y Educación de la Universidad Distrital Francisco José de Caldas. Investigador del Grupo de Investigación Docimófilos. Correo electrónico: hsgonzalezm@udstrital.edu.co 
De una manera menos ambiciosa, en términos generales, se hará referencia en este artículo de reflexión a las "bondades" que implica la posibilidad de una perspectiva de formación investigativa en la universidad, en oposición a un enfoque tradicional de la educación, en la medida en que el fortalecimiento de una docencia que se base en la implementación de procesos investigativos aportaría ampliamente a la formación pedagógica de los estudiantes, dando sentido a las prácticas de enseñanza.

El mundo globalizado, regido por estándares en todos los ámbitos, determina las formas y posibilidades de concebir la educación. En este sentido, Mario Díaz comenta: "Hoy las investigaciones deben girar alrededor de la práctica pedagógica, la construcción del saber pedagógico, la construcción de identidades académicas y profesionales que proyectan nuevos discursos y prácticas en una sociedad dominada cada vez mas por los signos de globalización" (Díaz, 2000). Así, es clave reconocer también el sentido de la investigación local en cuanto permite establecer un contraste con lo global y la posibilidad de un aporte sustantivo a la comunidad académica internacional.

\section{Universidad y conocimiento}

La universidad es sin duda el espacio de la creación, el lugar donde se piensan, se reflexionan y se analizan las temáticas que atañen la vida de las sociedades en las cuales se inscriben. Como bien lo dice Mario Díaz en su análisis sobre la formación de profesores en Colombia: "La universidad y en general, el sistema de educación superior en su conjunto, pueden entenderse como instancias de producción y reproducción de la cultura en sus diferentes manifestaciones" (Díaz, 2000: 20). Esto significa que la universidad está constituida por una red de relaciones internas y que atienden la demanda de una sociedad determinada, lo que hace del caso colombiano una importante oportunidad de salida a la encrucijada actual.

En este orden de ideas, es importante decir que el problema de la producción de conocimientos se relaciona directamente con la constitución, circulación y enseñanza de las diversas disciplinas del conocimiento que generan la posibilidad de creación de nuevos conocimientos. De esta manera, los procesos de investigación se constituyen en una nueva manera de dar cuenta del aprendizaje $y$ en general de relacionarse con el conocimiento en cuanto forma de producción, lo que contrasta con un escenario en el que la educación se asume como la simple posibilidad de acceder a un título que posibilita una forma laboral que da cuenta de la subsistencia individual sin la toma de conciencia de un empoderamiento colectivo que avanza hacia mejores formas de vida de la comunidad.

En la actualidad, el conocimiento es un problema de información en el que lo importante no es tanto cómo acceder al mismo sino cómo usarlo. En otras palabras, o producimos conocimiento o lo consumimos. De ahí la necesidad de fortalecer los procesos de investigación en la universidad, ya que a riesgo de no estar en la vanguardia de la producción de conocimiento, no nos quedaría otro remedio que el de ser gregarios del material producido en otras latitudes y contextos o, en el mejor de los casos, la vana resignación de adaptar dichas producciones a nuestras necesidades específicas.

\section{Consideraciones sobre la investigación en la educación superior}

La investigación en sí misma requiere en una primera instancia de una actitud y una capacidad de observación que genere unas acciones puntuales frente a los retos que ella misma genera. Como diría el maestro Estanislao: "Es necesario que se transforme algo en mi manera de sentir para conocer algo" (Zuleta, 1986). Lejos de lo que algunos imaginarios plantean, la investigación no es un privilegio para eruditos y/o superdotados sino un requerimiento básico para la supervivencia, avance y desarrollo de cualquier sociedad. En Colombia ha sido importante la investigación de carácter formativo, la cual integra las disciplinas propias del programa con la práctica pedagógica y la metodología de la investigación para resolver problemas de índole educativo, como servir de fundamento en la didáctica o insertarse en investigaciones de mayor alcance. "la investigación formativa constituye una estrategia pedagógica de carácter docente para el 
desarrollo del currículo" (Parra, 2004: 3). Este tipo de investigación permite implementar un aprendizaje autónomo y significativo. Su finalidad se centra en la apropiación de significados ya elaborados de los sujetos en formación. De esta manera, la investigación formativa y la investigación en "estricto sentido" se complementan en la medida en que la comunidad académica nacional e internacional las acepte y las valide como tal.

La investigación se orienta a la construcción de conocimiento sobre un objeto específico en el campo del saber y que pretende explicar algún fenómeno social o natural. En este contexto, la investigación presenta variados objetos de estudio que deben priorizarse en orden a las necesidades más sentidas de las comunidades. Así entendida la investigación no se limitará al mero diagnóstico de las problemáticas sino que intervendrá en la solución de los problemas que ha detectado y/o planteado.

En lo que respecta a la formación de educadores, la investigación tiene la posibilidad de constituirse en un procedimiento didáctico o praxis pedagógica que fortalezca la comprensión de la dinámica educativa y amplíe la visión y los alcances que genera el hecho de enseñar. Sin embargo, existe la creencia de que la investigación es para docentes universitarios de jerarquías diferentes, quienes le asignan a la investigación un papel de actividad especial complementaria a la formación básica. En este sentido, es pertinente recordar que la universidad enseña lo que una serie de estudios o investigaciones anteriores han concluido y que debe ser nuevamente a examen.

Se ha observado en Colombia en la última década, una mayor preocupación de las universidades por atar la formación inicial de los docentes a procesos de investigación formativa a través de los Semilleros de Investigación, la posibilidad de que los estudiantes se integren a los grupos de investigación constituidos por profesores universitarios y a los monitores de investigación. Estos procesos que buscan que los estudiantes realicen un acercamiento a la investigación todavía se encuentran en una fase incipiente, la cual es necesario fortalecer y masificar. Es importante que los procesos de forma- ción a través de la investigación lleguen a un mayor número de estudiantes, por esto es necesaria una renovación del currículo como tal.

La investigación educativa supone una percepción de la problemática educativa y unas formas de abordaje y solución de los problemas planteados que obligan a un manejo adecuado de las técnicas de investigación y a una toma de decisiones frente a la forma de "encarar" el objeto de estudio: la oposición o complementariedad entre el paradigma cuantitativo y el cualitativo, la validez interna en el planteamiento de los problemas de investigación, las metodologías de registro, la socialización de los resultados, etc. En este sentido, es importante anotar, como bien lo señala Guillermo Bustamante, que "El objetivo de la investigación no existe en bruto, lo que un investigador tiene delante de sí son ideas a través de las cuales se ve el objeto o gracias a las cuales no lo puede ver" (Bustamante, 1999).

\section{Algunas dificultades en la implementación de procesos investigativos en la educación superior}

A pesar de los ingentes esfuerzos en diversos sentidos y direcciones, hoy por hoy asistimos a dificultades originadas en una voluntad política todavía muy precaria en cuanto a la inversión económica necesaria en la medida que no se ha considerado como prioridad y para lograr garantizar los aportes necesarios los trámites burocráticos hacen difícil la gestión de los proyectos. Es recurrente observar en las universidades colombianas los siguientes problemas que interfieren en un adecuado desarrollo de la investigación:

- La falta de formación y de capacitación de los docentes en el campo investigativo.

- La saturación de asignaturas en los planes de estudio que no dejan espacio suficiente a los procesos formativos de investigación.

- La transmisión de conocimientos, en ocasiones desactualizados, no genera el desarrollo de formas que puedan producir nuevos conocimientos pedagógicos y/o didácticos. $\mathrm{Al}$ respecto, algunos estudios coinciden en que uno de los problemas que da lugar a las di- 
ficultades en la implementación de los procesos investigativos en la educación superior obedece al perfil del profesor, quien realiza principalmente una función de transmisor de contenidos, evidenciando prácticas tradicionales, que en algunos casos son reforzadas por los mismos estudiantes que reclaman estos métodos "ortodoxos" como evidencia del "buen docente".

- En ocasiones las líneas de investigación propuestas no se encuentran suficientemente articuladas a las problemáticas contextuales en las cuales se inscribe el saber pedagógico sobre el cual versa la investigación. Por esto es necesario generar una política de publicación de los resultados obtenidos que permita retroalimentar a las poblaciones objeto de las investigaciones de tal manera que se constituyan en una carta de navegación frente a la solución de los problemas abordados.

- Igualmente, la financiación de investigadores y la delimitación de campos considerados como prioritarios, atendiendo recomendaciones externas y concepciones poco comprometidas con la ejecución de una política equitativa en términos de cualificación de acuerdo a los diagnósticos que la misma comunidad académica ha generado.

- Aunque el auge de los posgrados ha representado un aumento de investigaciones y presencia de estudios cualificados dentro de la universidad toda vez que requieren como requisito de estos, la presentación de un trabajo investigativo, no siempre es posible la sostenibilidad y el impacto de los procesos investigativos generados.

\section{Investigación y normatividad en la educación superior}

Aunque la normatividad en el tema de la política educativa se refiera reiteradamente a la investigación, no la define ni contextualiza suficientemente, introduciéndola de forma abrupta y solicitando su aplicación desde un supuesto no desarrollado de la mejor forma. Por ejemplo, el artículo 109 de la Ley
General de Educación, que establece las finalidades de la formación de los educadores colombianos, plantea en su literal C: "fortalecer la investigación en el campo pedagógico y en el saber específico" (MEN, Ley General de Educación).

$\mathrm{Al}$ proponer la investigación pedagógica con una intencionalidad direccionada hacia la cualificación de docentes se pretende cualificar procesos de formación docente. En este sentido, los procesos de control estatal a partir de lo planteado por el CNA, en cuanto a acreditación universitaria, buscan un desarrollo de la investigación a partir de la presencia de doctores dentro de la planta de profesores, para que aporten desde su experiencia a los programas, departamentos y facultades a las que pertenecen. Aunque esta medida no garantiza necesariamente que se dé un desarrollo adecuado de los procesos formativos de investigación.

\section{La relación docencia - investigación}

Es importante superar la tradicional división entre docencia e investigación, que genera una escisión que dificulta los procesos de formación investigativa. En palabras de Mario Díaz, "La división del trabajo académico reproduce una oposición entre docencia (enseñanza, investigación y extensión). La estratificación de estas prácticas ha generado un aislamiento muy fuerte entre la docencia y la investigación...la investigación transmite un valor y un potencial académico superior al de la docencia: La investigación moviliza recursos" (Díaz, 2000: 22). Este sentido de superioridad asignado a la investigación genera distancias con la docencia que dificultan la integración entre ambas instancias que se complementan. "El aislamiento de la investigación de la docencia reproduce la distribución de percepciones, de conocimientos y de posibilidades de sus agentes, los profesores" (2000: 20).

La investigación sobre la enseñanza de su disciplina genera cambios en la práctica tanto de los docentes universitarios como de los estudiantes en formación. De hecho, la reflexión sobre la práctica pedagógica o sobre las creencias, imaginarios o representaciones que los docentes mantienen sobre 
determinados temas o problemas se asume como un importante insumo que necesariamente transforma efectivamente la práctica pedagógica.

A continuación se plantearán algunos elementos a tener en cuenta para el direccionamiento de la investigación por parte de los docentes:

- Investigar se aprende investigando y en este sentido de lo que se trata es de abrir los espacios desde la cátedra y el currículo para ser consecuentes con este postulado.

- Pertinencia de los problemas de investigación con el referente contextual.

- La investigación se constituye en una forma de enfrentar al estudiante con situaciones y experiencias que enseñan maneras de construir el pensamiento.

- El estudiante que experimenta y que aprende hace lo mismo que el sabio que investiga, tiene un fin que desea alcanzar, sigue un camino, emplea los procedimientos de la investigación, pone en actividad capacidades para lograr expresar los resultados a los que llega.

- Quien no aprendió a investigar, en el sentido de crear y recrear el conocimiento en su proceso formativo, solo podrá enfrentarse a problemas definidos desde lugares comunes pero nunca en lo específico de cada situación concreta. Por eso, es importante desarrollar las habilidades y las actitudes del aprendizaje durante toda la vida, elemento indispensable para hacer frente a las dificultades cotidianas que entraña el ejercicio profesional. Es importante formar con la convicción de que el estudiante ya es un agente de cambio y, por lo tanto, es un transformador activo dentro de la sociedad.

- La mejor manera de enfrentar con solvencia los problemas de la práctica profesional se propone a partir de la investigación. El estudiante en sus procesos de formación aprende a indagar en diversas fuentes de información a la vez que realiza ejercicio formativos en los componentes, asignaturas o cursos, paralelamente realiza trabajos, reportes o ensayos en términos de resolución de problemas. El profesor es el protagonista clave en la implementación de estos procesos, él conduce a fuentes probables de conocimiento, y es necesaria su experiencia investigativa y su actitud de apertura frente al conocimiento en general. Por otra parte, es importante que el profesor aprenda a la vez que enseñe y el alumno enseñe a la vez que aprenda.

- El aprendizaje desde el estudiante se basará en la lógica de la construcción del conocimiento que será descubierto por el profesor en la medida en que propicie las condiciones de transformación de su propia práctica docente.

- La intención más importante reside en descubrir los alcances, las aptitudes y las posibilidades que poseen los estudiantes para que estas sean desarrolladas en su quehacer personal y profesional. En este sentido, Bruner afirma que el primer objetivo de cualquier acto de aprendizaje además del placer es que este sea útil para el futuro (Bruner, 1963).

- Es importante señalar el papel protagónico asignado aquí al estudiante en la medida que la disciplina del trabajo académico, la práctica de observación, los procesos de pensamiento superior relacional e inferencial son estimulados al igual que el rigor metodológico, la claridad expositiva y el manejo de herramientas de investigación.

Una docencia basada en la investigación puede tener ciertas resistencias, en la medida en que genera procesos pedagógicos de innovación que a su vez requieren de una suficiente ilustración para ser aceptados, a la vez que contradicen el paradigma tradicional. Volviendo a la máxima de "el hombre es un animal de costumbre", los mismos estudiantes muchas veces prefieren que se les entregue todo bien digerido al planteamiento de las búsquedas y al reto de acercarse a problemas concisos de difícil manejo. El mismo cambio en la relación del conocimiento de una manera más horizontal puede producir un cierto desequilibrio en la medida que en cambio de repetir, invita a pensar y a cuestionar desde el propio punto de vista y a partir de los resultados obtenidos en las indagaciones de los estudiantes. 


\section{Propuestas para la cualificación de la investigación en la educación superior}

Los procesos investigativos en la educación superior no deben relegarse a una formación complementaria a la que solo pueden acceder algunos estudiantes interesados y con unas condiciones específicas. La formación pedagógica se debe realizar teniendo como eje principal del currículo los procesos de investigación a través de acompañamiento por parte de los docentes en las diferentes fases de enunciación, desarrollo y sistematización de los proyectos de investigación. Esto permitirá dar cuenta del desarrollo de los proyectos, un trabajo práctico en investigación, acompañado de un rastreo de problemas específicos a partir de las metodologías de investigación que contribuirán, de manera fundamental, a la motivación y a la manera como el estudiante podrá constituir su propio proceso de formación desde la investigación, de tal forma que pueda dar cuenta de un acercamiento a la perspectiva educativa a partir de la formulación y de la ejecución de proyectos que fortalecen su formación como docente.

La realización de esta propuesta implica una nueva mirada de lo curricular, a la vez que un fortalecimiento en las prácticas de los docentes, quienes realizarán el acompañamiento desde la cátedra pensada como investigación. En este sentido, paradójicamente se observa que asignaturas como Metodología de la Investigación pueden ser impartidas por docentes que no poseen experiencia investigativa. La experiencia del docente universitario en el campo investigativo se reduce por lo general a su trabajo de grado (Tamayo, 1999).

Es importante tener en cuenta que las reformas curriculares conllevan cambios en las conceptualizaciones de los profesores que las llevan a cabo, esto desafortunadamente no implica que se realicen cambios en sus acciones docentes.

Además de implementar currículos que privilegien la investigación como proceso de formación, es necesario implementar la interacción de los procesos de investigación de forma continua en el quehacer cotidiano, es decir, la implementación de una cultura de la investigación en la misma práctica docente, a través de la síntesis y retroalimentación entre la docencia y la investigación.

\section{Síntesis conclusiva}

En el presente artículo se plantea la importancia de la investigación en la educación superior, destacando el papel que la investigación formativa ha generado en el sentido de una aproximación sugestiva al hecho de investigar. Por otra parte, se señala la tensión entre docencia e investigación, dando a conocer las dificultades que se ciernen sobre esta. Se han delineado algunos elementos de acción y reflexión que contribuyen a la cualificación de los procesos de investigación en la universidad.

La implementación de estos procesos que se pueden llevar a cabo por un estudiante responsable, crítico y creativo será posible solo con una voluntad política que articule la institucionalidad de la educación superior a los procesos de investigación desde una perspectiva justa donde las políticas de contratación, cualificación y profesionalización de la docencia generen las expectativas aquí previstas.

\section{Bibliografía}

Álvarez Salgado, E., et al. (S.F.). Licenciatura en Biología. Proyecto curricular. Facultad de Ciencia y Tecnología. Bogotá: Universidad Pedagógica Nacional.

Bruner, J. (1963). El proceso de la educación. México: Uteha.

Díaz Villa, M. (2000). La formación de profesores en la educación superior Colombiana: problemas, conceptos, politicas y estrategias. Instituto Colombiano para el fomento de la Educación Superior ICFES. Bogotá, 2000.

\section{Artículos}

Álvarez, A. (1997). "Pensar la universidad desde la investigación”. En Pedagogía y Saberes, núm.10. Bogotá: Universidad Pedagógica Nacional. 
Bustamante, G. (1999). "Algunos elementos para pensar la investigación educativa”. En Pedagogía y Saberes, núm. 13. Bogotá: Universidad Pedagógica Nacional.

Morán, P. (1993). "La Vinculación Docencia - Investigación como Estrategia Pedagógica". En Perfiles Educativos, núm. 61. México: Universidad Nacional Autónoma de México.

Parra, C. (2004). "Apuntes sobre la investigación formativa”. En Educación y Educadores, vol. 7. Bogotá: Universidad de la Sabana.
Tamayo, A. (1999). "La investigación en educación y pedagogía en Colombia”. En Pedagogía y Saberes, núm. 13. Bogotá: Universidad Pedagógica Nacional.

Zuleta, E. (1986). “Teorías freudianas de la infancia”. En Boletín de estudios Psicoanalíticos, vol. 1. núm. 1. Cali. 